\title{
Bioceramic Material Stimulation Affects Perceptual Consciousness: A Study Combining Descriptive Observation and Functional MRI Connectivity Analysis
}

\author{
Ting-Kai Leung \\ Taoyuan General Hospital, Ministry of Health and Welfare \\ Chia-Wei Li \\ Taipei Medical University \\ Yu-Chun Lo \\ Taipei Medical University \\ Ping-Yen Tsai \\ Taipei Medical University \\ Jia-Yi Wang ( $\sim$ jywang2010@tmu.edu.tw ) \\ Taipei Medical University
}

\section{Research Article}

Keywords:

Posted Date: January 14th, 2022

DOI: https://doi.org/10.21203/rs.3.rs-1199767/v1

License: (c) (i) This work is licensed under a Creative Commons Attribution 4.0 International License.

Read Full License 


\section{Abstract}

There is still no clear explanation of the process of perceptual consciousness that connects our body with brain. Innovation on the technology of bioceramic has now advanced towards clinical applications, including rehabilitation of brain infarction, therapies of insomnia and migraine. To demonstrate how 'resonant energy transfer through the bioceramic material with tempo sound and visible light spectrum' (bioceramic material stimulation, BMS) non-invasively affects perceptual consciousness, we investigated the responses of participants to BMS on perceptual consciousness by questionnaire of subjective descriptions and analyzed resting state fMRI during BMS. There were $61.3 \%$ participants who were categorized as positive group with various types of perceptual consciousness. By setting a threshold value at ' $p<0.001$ ', enhanced connections of 'parahippocampal gyrus to cerebellar lobule $\mathrm{V}$ ' and 'angular gyrus to precuneus' were found. However, decreased connection of 'caudate nucleus to cerebellar lobule VIIb' was found. We conclude that the most affected brain functions by BMS including somatosensory, audio-visual perception and social cognition. The analysis of functional connectivity during BMS may help us gain more knowledge of consciousness and related division of neuroscience in humans.

\section{Introduction}

To date, there is no clear explanatory link connecting 'consciousness' to the human body and brain. Thus, many studies have been designed to account for subjective conscious experiences in terms of different features of brain states and neural interactions ${ }^{1}$. Current knowledge of neurophysiological approaches revealed that different states of consciousness are mainly brought about by a compromised brain anatomy, transient changes in connectivity, and neurochemical and metabolic processes. Altered states of consciousness could be evoked by: (1) physical and physiological stimulation (such as transcranial brain stimulation) ${ }^{2}$; (2) chemical drugs (such as alcohol and hallucinogen) ${ }^{3}$; (3) psychological means (such as hypnosis) ${ }^{4}$; (4) physical diseases (such as sleep deprivation or brain tumor) ${ }^{5}$; and (5) spontaneously (mythology or religion) ${ }^{6,7}$.

To investigate different state of consciousness noninvasively, we used BMS to generate biological field in this study. The advantage of BMS as based on the characteristics of bioceramic material which emits non-ionized radiation that can be amplified by sound. It produces a weak biological field effect and has been hypothesized as a new concept in physics ${ }^{8,9}$. Research results show remarkable effects both physically and physiologically. Weakening of hydrogen bonds, improved microcirculation, better absorption of water-treated with bioceramic material, speeding up the healing mechanisms in tissue damage, enhanced plant growth, and many more physical-biological effects ${ }^{10-14}$ have been observed in the laboratory and preliminary clinical trials ${ }^{15-17}$. In our previous research, BMS treatment significantly improved sleep quality in insomnia patients ${ }^{18}$. BMS causes a significant elevation in the beta spectrum of the EEG (at 15-27 Hz), in contrast to non-BMS group of participants ${ }^{18}$. Our clinical studies have shown that BMS attenuated symptoms in somatization disorder, obsessive-compulsive disorder, interpersonal sensitivity, schizophrenia, depression and anxiety ${ }^{19}$. 
In the present study we examined whether BMS enhanced various types of perception through certain parts of the body with no actual direct physical stimulation to the corresponding parts of the sense organs. We have designed a questionnaire in this study based on a previous research on electrical stimulation of the human brain induced various sensations and defined it as a 'perceptual and behavioral phenomena' ${ }^{2}$. It is suggested that the perceptual consciousness are beyond the usual five senses of external stimulation by sight, sound, smell, taste, and touch. In other words, there is no direct stimulation of the peripheral nervous receptors on the sensory organs of tongue, eye, skin, nose, and ear, but it is sensed by the central nervous system or mind that may be related to high level of consciousness ${ }^{3,20}$. In our previous study, activation of various brain areas during BMS were detected by $\mathrm{fMRI}^{18}$. Moreover, during BMS participants that was monitored by blood oxygenation level dependent (BOLD) functional magnetic resonance imaging (fMRI) imaging, participants were recorded with increase activations in areas of the left temporal pole, left parahippocampal gyrus, left rolandic operculum, left inferior frontal gyrus, left precentral gyrus, left hippocampus, left middle frontal gyrus, left insula, left thalamus, and left cerebellum ${ }^{18}$. However, the functional connectivity between different brain areas during BMS was unknown. Through literature review, stimulating different locations of the human brain with specific parameters produced variability of the perceptual consciousness ${ }^{2,20}$.

In this study, we categorized the subjective responses of the participants with different types of 'perceptual consciousness' during BMS. In addition, resting-state fMRI was used to explore the possible activation of functional connectivity in the human brain during BMS.

\section{Methods}

\section{Bioceramic material stimulation (BMS)}

The bioceramic powder used in this study (obtained from the Bioenergy laboratory, Taoyuan, Taiwan) was composed of micro-sized particles produced from several ingredients mainly different elemental oxides. The $10 \%$ bioceramic powder ${ }^{13,16}$, mixed with silicone rubber, produces sheet membrane with characteristics of spongy density (capable of sound transmission). Bioceramic is a kind of material with an energy field that can be enhanced by sound waves and visible light spectrum ${ }^{18,19}$. Bioceramic treatment also facilitates the breakup of large clusters of water molecules by weakening the hydrogen bond; this is one of the key mechanisms of bioceramic treatment on biological-physical and physicalchemical processes ${ }^{10-12}$. In this study, two types of BMS were used, including (i) For questionnaire assessment: participates lying on a platform with head contact with bioceramic silicon rubber sheet enhanced by a tempo sound system setting at $1-12 \mathrm{~Hz}$ with about $70 \mathrm{~dB}$ and visible light spectrum range from 400 to $760 \mathrm{~nm}$ with level of illumination at about 200 lux. ${ }^{21}$ (Figure 1) and (ii) For fMRI analysis: participates lying on MRI scanner with bioceramic silicon rubber sheet placed on bilateral temporal scalp regions, which enhanced by acoustic noise of pulse sequence at about $100 \mathrm{~dB}$ during fMRI imaging ${ }^{22}$. All methods were carried out in accordance with relevant guidelines and regulations 


\section{Study participants.}

The human volunteers that participated in the clinical trials were sourced using poster advertisements placed on bulletin boards near practice waiting rooms. There were 155 (female: 91; male: 64) volunteers. Participants were screened and none were found with neurological/psychiatric disorders, any indications of drug abuse, or sleep deprivation. The study was conducted at the Taoyuan General Hospital, Taiwan and the Human Study protocol was approved by the Medical Ethics and Institutional Review Board of the Taoyuan General Hospital, Ministry of Health and Welfare,Taiwan (approval no.: TYGH106015, see APPENDIX 1). Prior to participating in this study, the participants were all required to sign informed consent forms (see APPENDIX 2).

\section{Questionnaire Assessment Of Subjective Descriptions Of The Bms}

In the beginning, volunteer participants sat alone in the experimental room for about 5 minutes (as control condition). Then, the control and the experimental groups received the experiment protocol using the usual sound and BMS system for 20 minutes, respectively. Participants were asked to pay attention and record any change and all sensations that they may experience before and after the BMS xperiment. A questionnaire (Table 1) was designed to quantify the different types of 'perceptual consciousness' (level 0-3) by referring to a previous brain stimulation research ${ }^{2}$ that our participants experienced in their subjective response to the BMS system. The participants were then categorized as positive for 'perceptual consciousness' if they belonged to levels 1 to 3 (Table 1 ).

\section{fMRI data acquisition and preprocessing.}

With reference to the previous resting state fMRI study ${ }^{23}$, our protocol was designed by the following three steps: (1) MRI acquisition and preprocessing; (2) Network construction and functional module parcellation graph construction; and (3) Differences in effects without and with BMS on the functional connectivity of the brain.

For imaging data collection, the participants were scanned using a 1.5T Philips MRI/MRS system with a 20-channels array head coil. Resting state fMRI involved generating a series of $4 \mathrm{~mm}$ axial slices of the region of interest which were acquired using a gradient echo planar imaging (EPI) with the following parameters: time to repetition $=2500 \mathrm{~ms}$, echo time $=30 \mathrm{~ms}$, flip angle $=78^{\circ}$, in-plane field of view $=210 \times$ $210 \mathrm{~mm}$, and acquisition matrix $=64 \times 64 \times 26$ to cover whole cerebrum. After all the resting state fMRI scans, one turbofield echo (TFE) 3D T1-weighted imaging (TR/TE $=7.468 / 3.453 \mathrm{~ms}$ ) with spatial resolution of $0.64 \times 0.64 \times 1.2 \mathrm{~mm}^{3}$ was acquired.

The first five volumes were discarded to allow the magnetization to approach a dynamic equilibrium. The preprocessing of images was performed with SPM12 (Wellcome Trust Centre for Neuroimaging; http://www.fil.ion.ucl.ac.uk/spm) and Resting-State fMRI Data Analysis Toolkit (REST) ${ }^{24}$. For motion 
correction, aligning each volume to a reference base volume was performed across the functional dataset. Each functional dataset was filtered using a low-pass Chebyshev Type II filter with a frequency range of 0 to $0.1 \mathrm{~Hz}$ (in MATLAB; MathWorks, Inc., Natick, MA, USA). After filtering, linear trends were removed to eliminate signal drift induced by system instability. The individual functional images were normalized to the corresponding Montreal Neurological Institute (MNI) space by applying transferred parameters, which calculated from the whole-brain-coverage EPI images and MNI template, and linearly resampled to an isotropic resolution $(2 \times 2 \times 2 \mathrm{~mm} 3)$. Finally, all datasets were smoothed using a $6 \mathrm{~mm}$ FWHM Gaussian kernel to minimize inter-individual variances and to enhance SNR.

\section{Analysis for functional connectivity.}

To objectively and comprehensively study the fluctuations in brain activity during BMS, we computed the regional pairwise correlation coefficients (RPCC) between the fMRI activity from ROls selected based on the 'Automated Anatomical Labeling' (AAL) map template ${ }^{25}$. Altogether, 116 brain regions were used as node ROIs in the RPCC analysis, and each pixel of RPCC matrices shows the correlation between a node $\mathrm{ROI}$ and the other one. The MATLAB software was used in all the data analysis and result presenting.

\section{Results}

\section{Outcome based on various types of 'perceptual consciousness'}

There were 155 questionnaires $(n=155)$ gathered for the experiments using the $B M S$, and we found that $60(38.7 \%)$ participants were categorized as "none" perceptual consciousness and belonged to the negative group. The remaining $95(61.3 \%)$ participants were positive group and categorized as various perceptual consciousness types (described in Table 1) such as 73 (47.1\%) with cutaneous, $13(8.4 \%)$ with motion or rotation, $8(5.2 \%)$ with visual audio-visual and $1(0.6 \%)$ with social cognition type of perceptual consciousness (Figure 2). By using 'the Chi Square Goodness of fit' test, a significant statistical difference was found between negative and positive groups (as $p<0.05^{\star}$ ).

\section{Analysis of Brain Connectivity Networks.}

After using the regional pairwise correlation coefficients (RPCC) method for variation of resting-state fMRI study between without BMS and with BMS on the 18 participants, it was then compared with the differences in brain connectivity of networks of without BMS and with BMS (Figure 3). By setting a threshold value at ' $p<0.001$ ', enhanced connections of 'parahippocampal gyrus to cerebellar lobule $V$ ' and 'angular gyrus to precuneus' were found. On the contrary, decreased connection of 'caudate nucleus to cerebellar lobule VIIb' was found (Figure 4).

\section{Discussion}




\section{Comparison with direct electrical stimulation of the brain.}

Comparing our results of bioceramic treatment with direct electrical stimulation of the brain ${ }^{2}$, we found that some forms of subjective experiential phenomena and behavioral changes evoked by both direct electrical stimulation of the brain and BMS are similar: auditory and visual hallucinations; feeling of being somewhere else or even someone else; dream-like states; gustatory sensations; vestibular effects; feeling of unreality; recitation of lyrics and singing; simple movements; oroalimentary automatisms; epigastric sensations, nausea, urge to cry, and motor response (e.g. locomotion, eye and head turning, body swinging, olfactory sensation and thrusting) ${ }^{2}$. Although the effect of BMS as a technique for induction of perceptual consciousness is weaker than direct electrical stimulation of the brain, it has the advantage of being non-invasive and is free from clinical risk.

\section{Functional connectivity of BMS effect on neuropsychiatric problems.}

Our previous preclinical or clinical studies on BMS included positive outcomes of migraine ${ }^{17}$, rehabilitation of brain infarction ${ }^{14}$, autonomous nervous system disorders, and insomnia ${ }^{18,19}$. With respect to mental illness, BMS has proved its achievements of alleviating the drug withdrawal symptoms of amphetamine and hypnotic-drug addictions ${ }^{19}$. There were also reported cases of clinical improvement from psychological illness of depression ${ }^{19}$, anxiety, schizophrenia ${ }^{19}$ and suicidal attempts ${ }^{19}$.

Our study on BMS showed positive functional connectivity of resting state fMRI including 'parahippocampal gyrus to cerebellar lobule $\mathrm{V}$ ' and 'angular gyrus to precuneus' and negative functional connectivity on 'caudate nucleus to cerebellar lobule VIIb'. It has been reported that finger tapping (movement) activated cerebellar lobules IV-V and VIII. In addition to motor function, cerebellar lobules VI and $\mathrm{VII}$ is involved in more cognitively demanding task ${ }^{26}$. Also, caudate nucleus is engaged in numerous functions apart from movements ${ }^{27}$, including procedural learning ${ }^{27}$, associative learning ${ }^{28}$, inhibitory control of action ${ }^{27}$, reward functions ${ }^{29}$. Therefore, altered functional connectivity between these brain regions may affect cognition as well as motor function.

Combining our previous study on BOLD fMRI ${ }^{18}$ and the present study, the change in brain area activation and connectivity during BMS of the brain detected by $\mathrm{fMRI}$ with the corresponding brain function of anatomy, is shown in the table. Based on neuro-anatomical analysis, we speculate the most affected brain functions include memory retrieval, language and speech, social cognition, and affection and attention.

To interpret our results of resting-state fMRI, we further reviewed literature and found that positive functional connectivity on the parahippocampal gyrus, angular gyrus, and precuneus, are related to status of depression ${ }^{30,31}$. Similar studies on patients with insomnia showed functional connectivity on the parahippocampal gyrus and precuneus ${ }^{32}$. Change in functional connectivity was also found on the parahippocampal gyrus, angular gyrus, and posterior lobe of cerebellum in young adult suicide attempter 33. In adolescents with internet gaming addiction, another fMRI study of functional connectivity had 
shown locations on the anterior lobe of cerebellum, precuneus and parahippocampus ${ }^{34}$. A study on drug addiction of a heroin-dependent individual showed location on the parahippocampal region and caudate nucleus ${ }^{35}$..

\section{The possible clinical application of resting-state fMRI and non-invasive brain stimulation.}

In the past, diagnosis of mental disorders was based on neuropsychological evaluations from subjective professional opinions of psychiatrist that lacked reliable biomarkers to monitor the response to treatment ${ }^{36}$. Comparison of the healthy control and the psychotic depression group by resting-state fMRI analysis showed that psychotic depression was consistently associated with significantly decreased specific functional connectivity ${ }^{37}$. Patients with psychological disorders such as schizophrenia, epilepsy, depression, autism, attention de『cit hyperactivity disorder (ADHD), and Alzheimer's disease, were also found to be associated with abnormal activity of fMRI-related brain connectivity network ${ }^{36}$. In the future, combined evidence-based study of fMRI with qualitative neuroanatomical detection and non-invasive BIOCERAMIC stimulation may be applied to abnormal functions related to memory retrieval, language and speech, social cognition, and affection and attention. In addition, this platform may also be applied to psychological disorders of insomnia, drug addictions, and psychological illness of depression, anxiety, schizophrenia and suicidal attempts.

\section{The limitations of this study.}

The limitation of this study is that it is a study with small number participants; however, it provides promising preliminary results not yet found in the literature. Second,

\section{Conclusion}

This study presented subjective observation data on BMS and achieved alteration of perceptual consciousness accordingly. Using resting state fMRI, it also found that BMS has an association with increased resting-state functional connectivity between parahippocampal gyrus to cerebellar lobule $\mathrm{V}^{\prime}$ and 'angular gyrus to precuneus', but decreased connectivity between 'caudate nucleus to cerebellar lobule VIIb'. These recent findings may help further research on BMS with a larger sample size and for potential clinical applications. BMS compared to direct electrical stimulation of brain, has the advantage of being non-invasive and is free from clinical risk.

\section{Declarations}

\section{Acknowledgements}

This study was supported by grants (MOHW109-HSO-M-211-000001 \& TYGH106054) from the Taoyuan General Hospital, Ministry of Health and Welfare, Taiwan to TKL and a grant (MOST 110-2314-B-038-106) from the Ministry of Science and Technology of Taiwan to JYW. Authors are also grateful to Prof. MingTse Lin on the assistance to this study. 


\section{Author contributions}

T.K.L. contributed substantially to study design, data interpretation and manuscript draft. C.W.L. performed the experiments and analyzed the data. Y.C.L analyzed data and edited P.Y.T searched the literature and edited the manuscript. J.Y.W contributed to the study design, data interpretation, manuscript draft, and manuscript revision. All authors reviewed the manuscript

\section{Competing interests}

The authors declare no conflict of interests.

\section{References}

1 Fazekas, P. \& Overgaard, M. Perceptual consciousness and cognitive access: an introduction. Philos Trans R Soc Lond B Biol Sci373, doi:10.1098/rstb.2017.0340 (2018).

2 Selimbeyoglu, A. \& Parvizi, J. Electrical stimulation of the human brain: perceptual and behavioral phenomena reported in the old and new literature. Front Hum Neurosci4, 46, doi:10.3389/fnhum.2010.00046 (2010).

3 Liechti, M. E., Dolder, P. C. \& Schmid, Y. Alterations of consciousness and mystical-type experiences after acute LSD in humans. Psychopharmacology (Berl)234, 1499-1510, doi:10.1007/s00213-016-4453-0 (2017).

4 Terhune, D. B., Cleeremans, A., Raz, A. \& Lynn, S. J. Hypnosis and top-down regulation of consciousness. Neurosci Biobehav Rev81, 59-74, doi:10.1016/j.neubiorev.2017.02.002 (2017).

5 Mason-Browne, N. L. Alteration of consciousness: tumor of the reticular activating system. AMA Arch Neurol Psychiatry76, 380-387, doi:10.1001/archneurpsyc.1956.02330280038004 (1956).

6 Clart, P. Spirit-Writing and the Cultural Construction of Chinese Spirit-Mediumship. Moral Mediums25, 153-189 (2003).

7 Charlton, B. G. Alienation, recovered animism and altered states of consciousness. Med Hypotheses68, 727-731, doi:10.1016/j.mehy.2006.11.004 (2007).

8 Movaffaghi, Z. \& Farsi, M. Biofield therapies: biophysical basis and biological regulations? Complement Ther Clin Pract15, 35-37, doi:10.1016/j.ctcp.2008.07.001 (2009).

9 Zhang, L. et al. Evaluation of Reflexology by "BIOCERAMIC Resonance" Operation producing Weak Force Field during Simultaneous Acupoint Stimulation of Urinary Bladder Point on Subject's Ear Resulting in Electric Current Change on Urinary Bladder reflex Point on Subject's Hands, and Related New Research Finding. Acupunct Electrother Res41, 207-224, doi:10.3727/036012917x14831065080096 (2016). 
10 Leung, T. K., Yang, J. C. \& Lin, Y. S. The Physical, Chemical and Biological Effects by Room Temperature Ceramic Far-infrared Ray Emitting Material Irradiated Water: A Pilot Study. J Chin Chem Soc59, 589-597 (2012).

11 Leung, T. K., Huang, P. J., Chen, Y. C. \& Lee, C. M. Physical-chemical Test Platform for Room Temperature, Far-infrared Ray Emitting Ceramic Materials (cFIR). J Chin Chem Soc58, 653-658 (2011).

12 Leung, T. K., Lin, S. L., Yang, T. S., Yang, J. C. \& Lin, Y. S. The Influence of Ceramic Far-Infrared Ray (cFIR) Irradiation on Water Hydrogen Bonding and its Related Chemo-physical Properties. Hydro/ Current Res5, 174 (2014).

13 Leung, T. K. In Vitro and In Vivo Studies of the Biological Effects of Bioceramic (a Material of Emitting High Performance Far-Infrared Ray) Irradiation. Chin J Physio/58, 147-155, doi:10.4077/CJP.2015.BAD294 (2015).

14 Zhang, L. et al. The Effect of Photoluminescence of Bioceramic Irradiation on Middle Cerebral Arterial Occlusion in Rats. Evid Based Complement Alternat Med2016, 7230962, doi:10.1155/2016/7230962 (2016).

15 Leung, T. K. et al. The Analysis of Normalized Effects on Meridian Current Level After the Photoluminescent Bioceramic Treatment on Acupuncture Points. J Altern Complement Med21, 472-479, doi:10.1089/acm.2014.0076 (2015).

16 Leung, T. K. et al. Bioceramic Resonance Effect on Meridian Channels: A Pilot Study. Evid Based Complement Alternat Med2015, 769546, doi:10.1155/2015/769546 (2015).

17 Chen, C. W. et al. Wave-induced flow in meridians demonstrated using photoluminescent bioceramic material on acupuncture points. Evid Based Complement Alternat Med2013, 739293, doi:10.1155/2013/739293 (2013).

18 Zhang, L. et al. A technology developed from concept of acupuncture and meridian system, the clinical effect of BIOCERAMIC resonance on psychological related sleep disturbance with findings on questionnaire, EEG and fMRI. J Tradit Complement Med8, 289-295, doi:10.1016/j.jtcme.2017.04.004 (2018).

19 Leung, T. K., Lee, C. M., Gasbarri, M. \& Chen, Y. C. Base on concept of traditional Chinese medicine: Experimental studies on efficacy of BIOCERAMIC Resonance to alleviate drug withdrawal symptoms. $J$ Tradit Complement Med9, 257-262, doi:10.1016/j.jtcme.2018.01.009 (2019).

20 Butler, A. B. Hallmarks of consciousness. Adv Exp Med Bio/739, 291-309, doi:10.1007/978-1-46141704-0_19 (2012).

21 Moseley, P., Alderson-Day, B., Ellison, A., Jardri, R. \& Fernyhough, C. Non-invasive Brain Stimulation and Auditory Verbal Hallucinations: New Techniques and Future Directions. Front Neurosci9, 515, 
doi:10.3389/fnins.2015.00515 (2015).

22 Price, D. L., De Wilde, J. P., Papadaki, A. M., Curran, J. S. \& Kitney, R. I. Investigation of acoustic noise on 15 MRI scanners from 0.2 T to 3 T. J Magn Reson Imaging13, 288-293, doi:10.1002/15222586(200102)13:2<288::aid-jmri1041>3.0.co;2-p (2001).

23 Kemmer, P. B., Guo, Y., Wang, Y. \& Pagnoni, G. Network-based characterization of brain functional connectivity in Zen practitioners. Front Psycho/6, 603, doi:10.3389/fpsyg.2015.00603 (2015).

24 Song, X. W. et al. REST: a toolkit for resting-state functional magnetic resonance imaging data processing. PLoS One6, e25031, doi:10.1371/journal.pone.0025031 (2011).

25 Tzourio-Mazoyer, N. et al. Automated anatomical labeling of activations in SPM using a macroscopic anatomical parcellation of the MNI MRI single-subject brain. Neuroimage15, 273-289, doi:10.1006/nimg.2001.0978 (2002).

26 Stoodley, C. J., Valera, E. M. \& Schmahmann, J. D. Functional topography of the cerebellum for motor and cognitive tasks: an fMRI study. Neuroimage59, 1560-1570, doi:10.1016/j.neuroimage.2011.08.065 (2012).

27 Nestler, E. J., Hyman, S. E. \& Malenka, R. C. Molecular neuropharmacology : a foundation for clinical neuroscience. 2nd edn, (McGraw-Hill Medical, 2009).

28 Anderson, B. A. et al. Linking dopaminergic reward signals to the development of attentional bias: A positron emission tomographic study. Neuroimage157, 27-33, doi:10.1016/j.neuroimage.2017.05.062 (2017).

29 Yager, L. M., Garcia, A. F., Wunsch, A. M. \& Ferguson, S. M. The ins and outs of the striatum: role in drug addiction. Neuroscience301, 529-541, doi:10.1016/j.neuroscience.2015.06.033 (2015).

30 Guo, W. et al. Abnormal resting-state cerebellar-cerebral functional connectivity in treatment-resistant depression and treatment sensitive depression. Prog Neuropsychopharmacol Biol Psychiatry44, 51-57, doi:10.1016/j.pnpbp.2013.01.010 (2013).

31 Leibenluft, E. \& Pine, D. S. Resting state functional connectivity and depression: in search of a bottom line. Biol Psychiatry74, 868-869, doi:10.1016/j.biopsych.2013.10.001 (2013).

$32 \mathrm{Li}, \mathrm{C}$. et al. Abnormal whole-brain functional connectivity in patients with primary insomnia. Neuropsychiatr Dis Treat13, 427-435, doi:10.2147/NDT.S128811 (2017).

$33 \mathrm{Cao}, \mathrm{J}$. et al. Abnormal regional homogeneity in young adult suicide attempters with no diagnosable psychiatric disorder: a resting state functional magnetic imaging study. Psychiatry Res231, 95-102, doi:10.1016/j.pscychresns.2014.10.011 (2015). 
34 Ding, W. N. et al. Altered default network resting-state functional connectivity in adolescents with Internet gaming addiction. PLoS One8, e59902, doi:10.1371/journal.pone.0059902 (2013).

35 Zhang, Y. et al. Distinct resting-state brain activities in heroin-dependent individuals. Brain Res1402, 46-53, doi:10.1016/j.brainres.2011.05.054 (2011).

36 Simon, R. \& Engstrom, M. The default mode network as a biomarker for monitoring the therapeutic effects of meditation. Front Psycho/6, 776, doi:10.3389/fpsyg.2015.00776 (2015).

37 Neufeld, N. H. et al. Resting state functional connectivity in patients with remitted psychotic depression: A multi-centre STOP-PD study. EBioMedicine36, 446-453, doi:10.1016/j.ebiom.2018.09.025 (2018).

\section{Tables}

Table1: Subjective descriptions of categories of perceptual consciousness induced by BMS

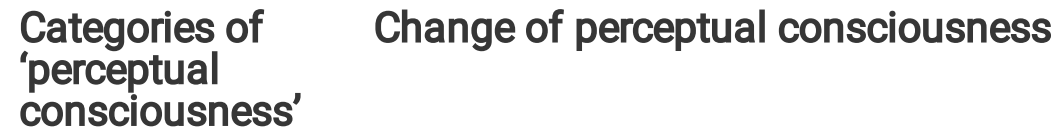

None
No significant change of 'perceptual consciousness'

Perception of the skin, such as increased vibration, itching, and slight inductance runs through the surface of the body (without real skin irritation)
Possible brain regions involved

None
Cutaneous

Motion or rotation
A deeper sense of motion or rotation, moving back and forth,

Somatosensory

Cortex

(Parietal cortex,Thalamus)
Visual or audiovisual phenomena
Visual phenomena such as seeing one or more specific colors or light (without real visual stimulation) More audio-visual phenomena, such as 'lucid dream like experience';
Social cognition 'sensation of consciousness leaving your body' feeling or 'interaction or communication with a person of consciousness', but candidates were left alone in the laboratory room.
Somatosensory Cortex

\section{Appendix}




\section{Figures}

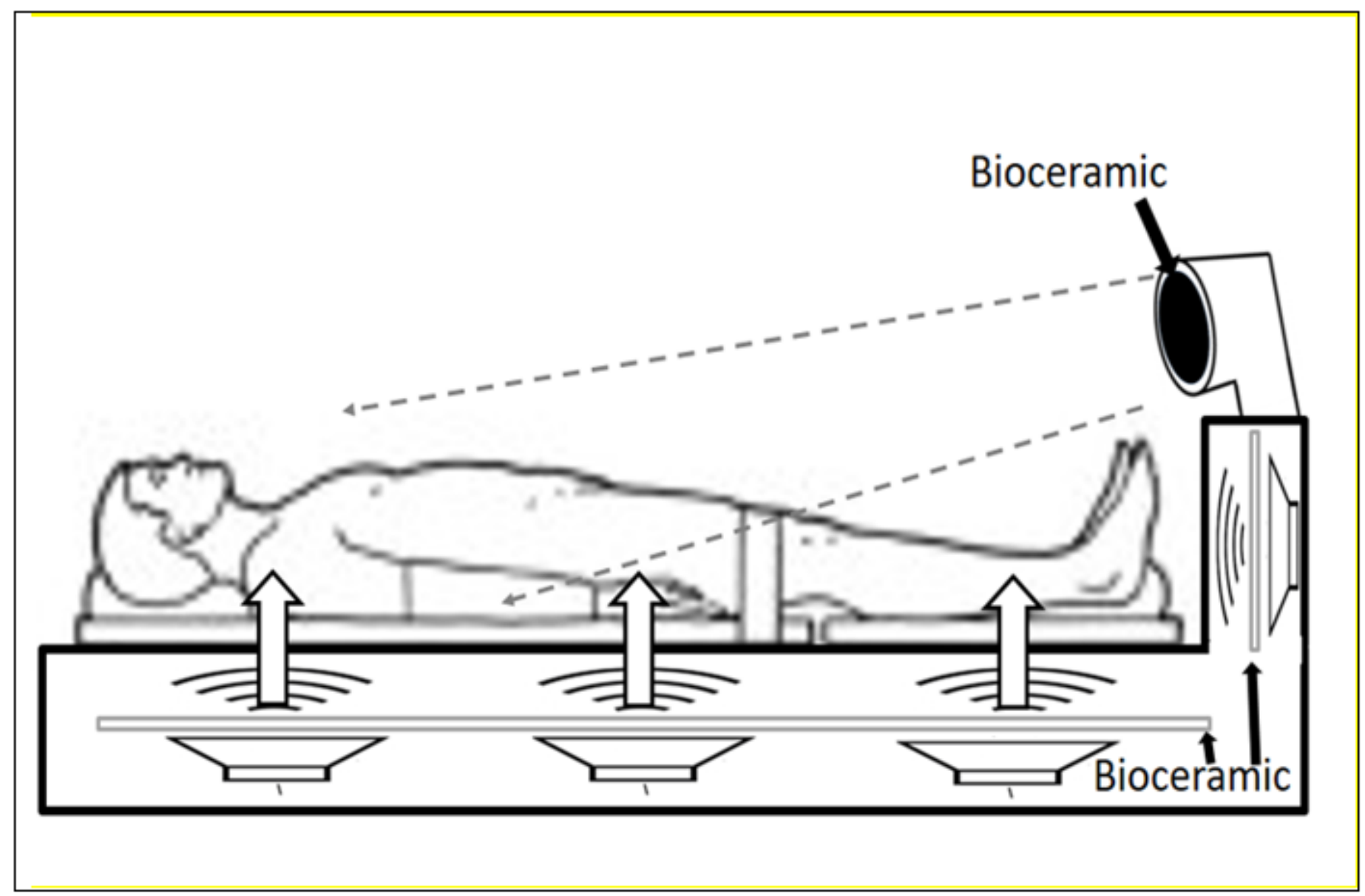

Figure 1

Conceptual drawing of bioceramic stimulation on each participant included resonant energy transfer through the bioceramic material with tempo sound (white arrows) and visible light spectrum (dotted black lines). 


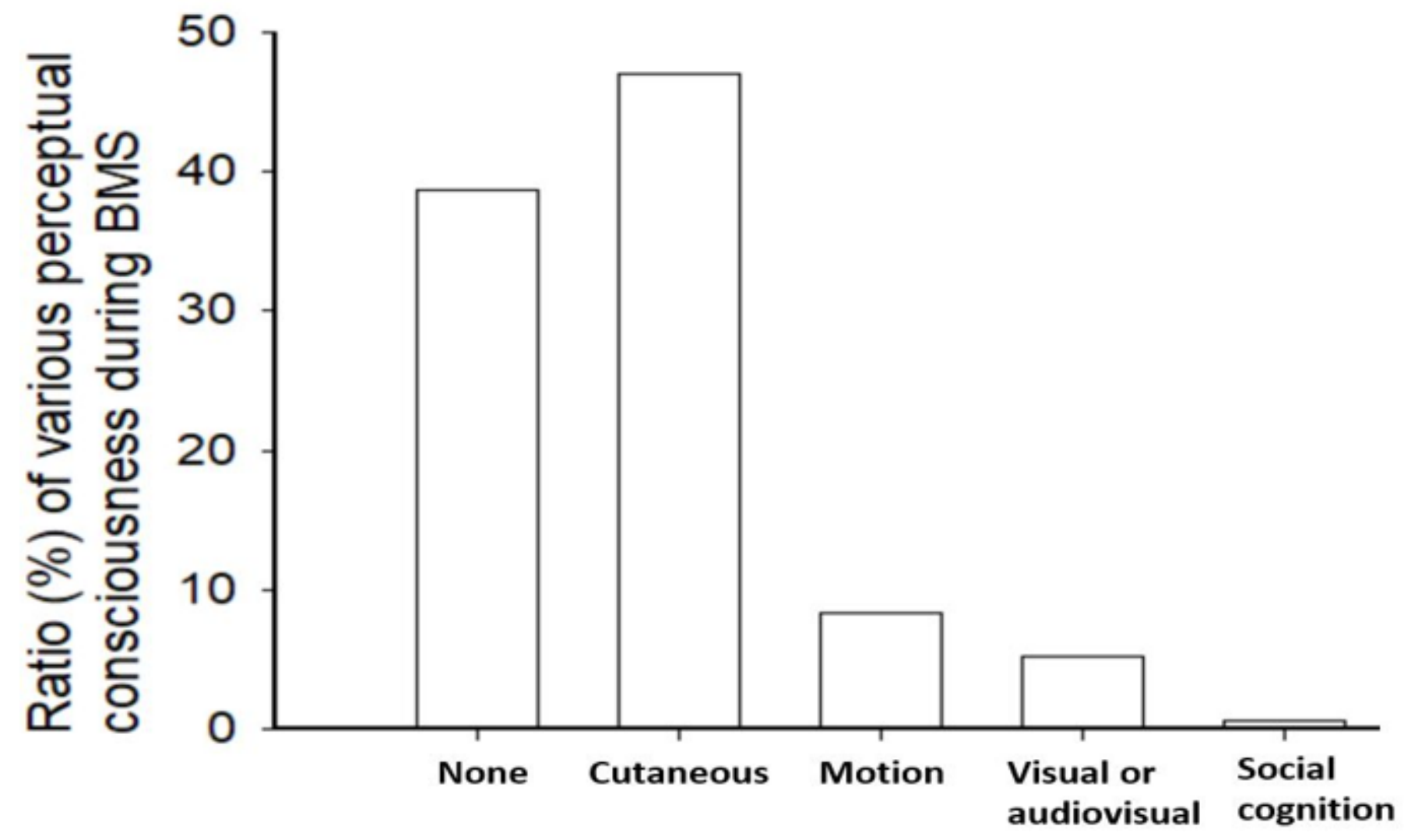

Figure 2

Ratio of various types of perceptual consciousness of subjective responses to BMS from 155 questionnaires 


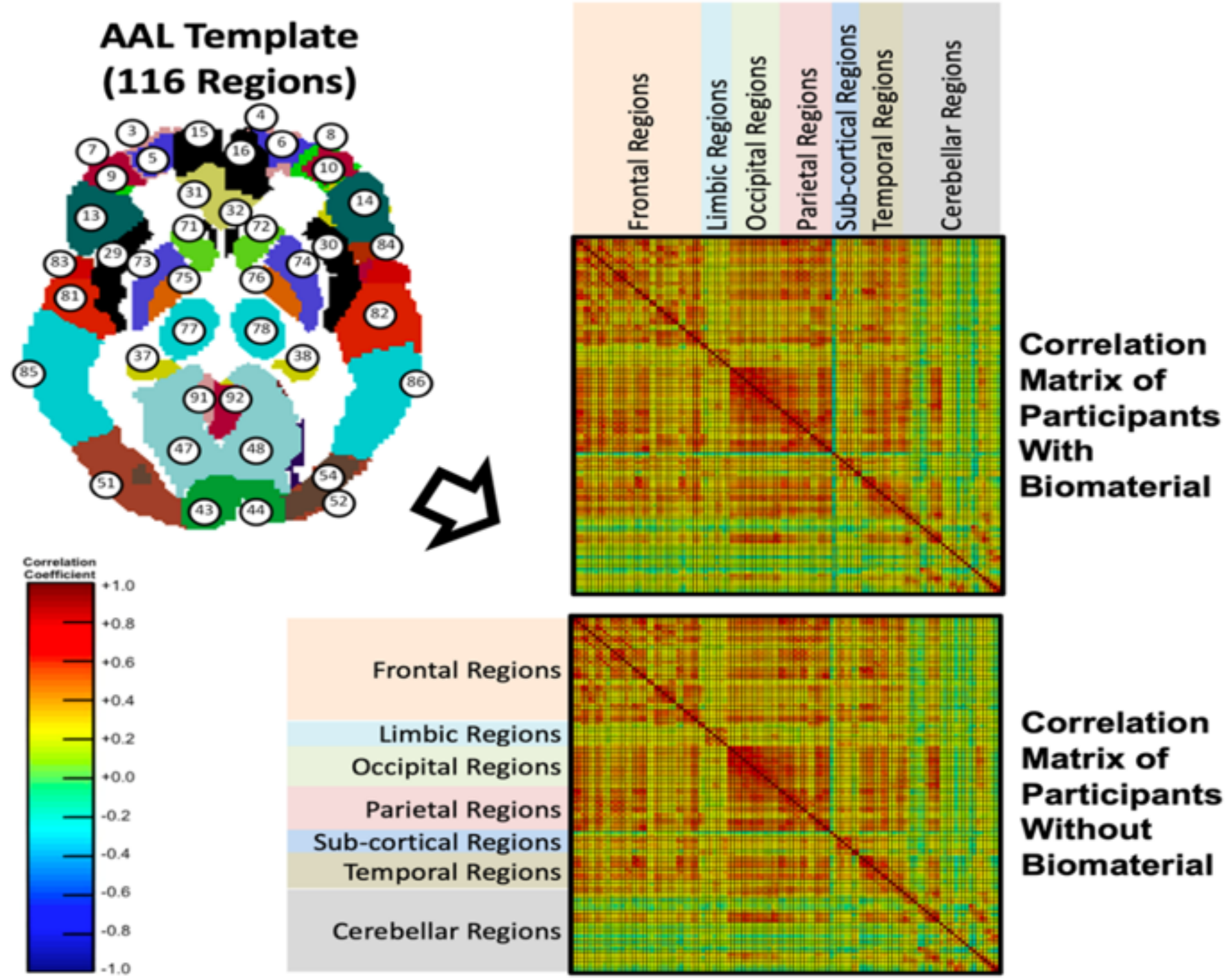

Figure 3

Edgewise connectivity matrices, averaged by subject group. The symmetric $116 \times 116$ connectivity matrices (as measured by Pearson correlations), averaged over the with/without BMS conditions. Edges are shown grouped by their module membership. Red edges indicate positive connectivity, while blue edges indicate negative connectivity.

AAL template, Automated Anatomical Labeling template. 


\section{Resting-State $_{\text {with Biomaterial }}>$ Resting-State without Biomaterial}

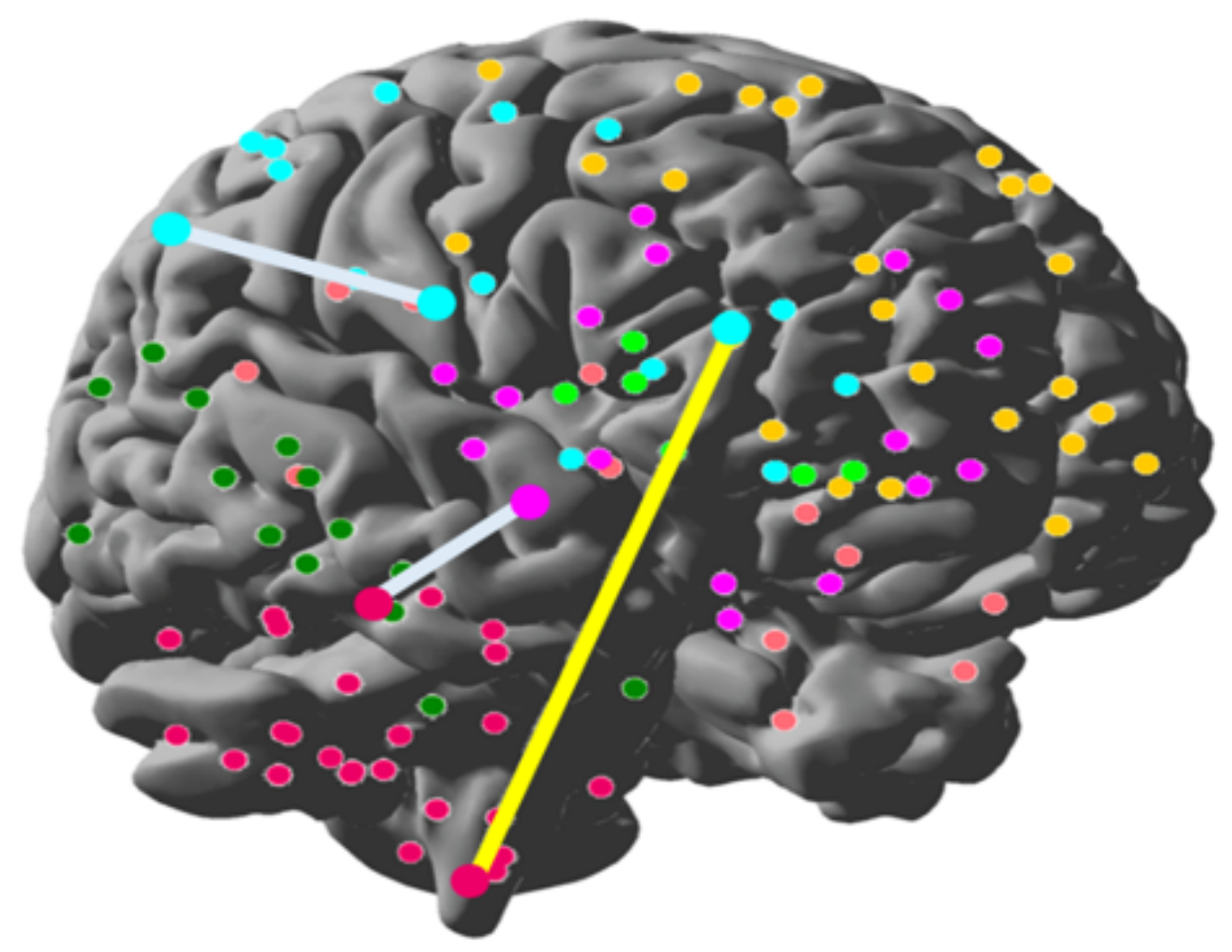

Parietal regions

Frontal regions

Sub-cortical regions

Limbic regions

Temporal regions

Occipital regions

Cerebellar regions

Figure 4

Positive (light blue) and negative (yellow) connections difference between without and with BMS. The threshold was $p<0.001$. 